\section{THE EFFECT OF RETROGRADE CEREBRAL PERFUSION AFTER PARTICULATE EMBOLIZATION TO THE BRAIN}

Neurologic injury as a consequence of cerebral embolism of either air or atherosclerotic debris during cardiac or aortic surgery is still a major cause of postoperative morbidity and mortality. While exploring various means of improving cerebral protection during complex cardiothoracic procedures, we have developed a chronic porcine model to study retrograde cerebral perfusion. We have previously demonstrated that retrograde perfusion results in a small amount of nutritive flow and provides cerebral protection that appears to be superior to simple prolonged hypothermic circulatory arrest. The current study was designed to evaluate the efficacy of retrograde cerebral perfusion in mitigating the effects of particulate cerebral embolism occurring during cardiac surgery. Four groups of pigs (19 to $28 \mathrm{~kg}$ ) underwent cardiopulmonary bypass with deep hypothermia at an esophageal temperature of $20^{\circ} \mathrm{C}$ : an antegrade control group ( $\mathrm{AC}, n=5$ ), an antegrade embolism group ( $\mathrm{AE}, \boldsymbol{n}=\mathbf{1 0}$ ), a retrograde control group ( $\mathrm{RC}$, $n=5$ ), and a retrograde embolism group ( $\mathrm{RE}, n=10$ ). In addition, because of extreme heterogeneity in outcome in the initial RE group, an additional group of 10 animals underwent embolism and retrograde perfusion at a later time. Embolization was accomplished by injection of $200 \mathrm{mg}$ of polystyrene microspheres ( 250 to $750 \mu \mathrm{g}$ in diameter) via the aortic cannula into an isolated aortic arch preparation in the $\mathrm{AE}$ and $\mathrm{RE}$ groups; the control groups received injections of $10 \mathrm{ml}$ of saline solution. After infusion of the microspheres or saline solution, conventional perfusion, with the aortic arch pressure maintained at $50 \mathrm{~mm} \mathrm{Hg}$, was continued for a total of 30 minutes in the antegrade groups; in the retrograde groups, retrograde flow was initiated via a cannula positioned in the superior vena cava, and was continued for 25 minutes. Superior vena caval flow was regulated to maintain a sagittal sinus pressure of approximately $30 \mathrm{~mm} \mathrm{Hg}$ in the retrograde groups, and blood returning to the isolated aortic arch was collected and measured. All animals were allowed to recover and were evaluated daily according to a quantitative behavioral score in which 9 indicates apparently complete normalcy, with lower numbers indicating various degrees of cerebral injury. At the time of planned death on day 6 , half of the brain was used for recovery of embolized microspheres after digestion with $10 \mathrm{~N}$ sodium hydroxide. The other half was submitted for histologic study. Neurologic recovery in

M. Enver Yerlioglu, MD (by invitation), David Wolfe, $\mathrm{MD}^{\mathrm{b}}$ (by invitation), Craig K. Mezrow, MS ${ }^{a}$ (by invitation), Donald J. Weisz, $\mathrm{PhD}^{\mathrm{c}}$ (by invitation), Peter S. Midulla, MD a (by invitation), Ning Zhang, MD (by invitation), Howard H. Shiang, DVM ${ }^{\text {a }}$ (by invitation), Carol Bodian, DrPh, ${ }^{\mathrm{d}}$ (by invitation), and Randall B. Griepp, MD, ${ }^{\mathrm{a}}$ New York, N.Y.
From the Departments of Cardiothoracic Surgery, ${ }^{a}$ Pathology, ${ }^{b}$ Neurosurgery, ${ }^{\mathrm{c}}$ and Biomathematical Sciences, ${ }^{\mathrm{d}}$ Mount Sinai Medical Center, New York, N.Y.

Supported by grant HL-45636-02 from the National Heart, Lung, and Blood Institute and in part by the Cardiothoracic Research Fund, Mount Sinai Medical Center, New York, N.Y.

Read at the Seventy-fifth Annual Meeting of The American Association for Thoracic Surgery, Boston, Mass., April 23-26, 1995.
Address for reprints: Randall B. Griepp, MD, Department of Cardiothoracic Surgery, Mount Sinai Medical Center, PO Box 1028, One Gustave L. Levy Place, New York, NY 10029.

J Thorac CARdiovasc SuRg 1995;110:1470-85

Copyright $(1995$ by Mosby-Year Book, Inc.

$0022-5223 / 95 \$ 5.00+0 \quad \mathbf{1 2 / 6 / 6 7 3 8 1}$ 
both the antegrade and retrograde control groups appeared to be complete, although mild evidence of histologic damage was present in some animals in the retrograde control group. After embolization, unequivocal neurologic injury occurred in both groups, accompanied by significant cerebral histopathologic abnormalities. Although neurologic outcome was not significantly better in the initial RE group as a whole than in the AE group, it was noted that several of the $R E$ animals recovered almost completely after retrograde cerebral perfusion (behavioral scores $>7$ ). The animals with good behavioral recovery were noted to have been perfused with markedly lower superior vena caval pressures than those used in animals that sustained severe neurologic injury. An additional 10 animals were therefore subjected to embolization and retrograde perfusion to clarify the impact on outcome of different superior vena caval pressures during retrograde perfusion. When these additional animals are included in the analysis, the behavioral and neuropathologic evidence suggests that use of retrograde cerebral perfusion may attenuate the severity of cerebral injury resulting from particulate emboli when adequate retrograde perfusion can be maintained at low superior vena caval pressures $(<40 \mathrm{~mm} \mathrm{Hg})$. This observation merits further study. (J Thorac Cardiovasc Surg 1995;110:1470-85)

$T_{\mathrm{m}}^{\mathrm{h}}$ he use of hypothermic circulatory arrest in the management of complex cardiovascular disease has achieved widespread clinical acceptance, but concern persists about its safety when longer durations of arrest are necessary. With highly complex operations requiring lengthy periods of hypothermic circulatory arrest now being carried out with increasing frequency, there is continuing interest in the study of methods that might improve cerebral safety in situations that require arrest of antegrade flow. Retrograde cerebral perfusion was proposed some years ago as a potential means of addressing this problem, and it is currently being explored in a number of ongoing studies as a means of improving cerebral protection by maintaining some degree of nutritive flow during hypothermic circulatory arrest. ${ }^{1}$

Although severe cerebral damage attributable to hypothermic circulatory arrest remains relatively uncommon, recent studies have confirmed the suspicion that subclinical cerebral injury occurs in a significant proportion of neonates after prolonged arrest, ${ }^{2,3}$ and that some degree of transient neurologic injury occurs in a substantial proportion of adult patients undergoing prolonged hypothermic arrest during aortic arch surgery. ${ }^{4}$ In addition, notwithstanding steady improvement in operative techniques and results, the small but significant number of patients in whom prolonged serious morbidity and mortality are directly attributable to cerebral dysfunction continues to be a concern, especially as the population undergoing cardiac operations both increases and ages.

Because most disabling cerebral dysfunction in adult patients after cardiac or aortic operations is due to focal injuries, ${ }^{4,5}$ strategies to avoid cerebral emboli have recently been emphasized in patients with extensive atheromatous disease of the aorta who are undergoing either aortic or coronary artery bypass operations. Therefore retrograde cerebral perfusion, in addition to its usefulness as an adjunct to hypothermic circulatory arrest, is attractive as a potential means of preventing air and particulate emboli, ${ }^{6,7}$ which are the major causes of permanent neurologic injury after cardiac and aortic operations in adults.

As part of an ongoing investigation of the usefulness of retrograde perfusion for cerebral protection in a chronic porcine model, this study was designed to explore the efficacy of an interval of retrograde cerebral perfusion in mitigating the potentially catastrophic effects of acute embolization during coronary or aortic operations. Our results suggest that retrograde perfusion may be useful in reducing cerebral injury under these circumstances.

\section{Materials and methods}

Preoperative management. All animals received humane care in accordance with the "Principles of Laboratory Animal Care" formulated by the National Society for Medical Research and the "Guide for the Care and Use of Laboratory Animals" prepared by the Institute of Laboratory Animal Resources and published by the National 
Table I. Mortality and behavioral outcomes

\begin{tabular}{crcc}
\hline Group & $n$ & $\begin{array}{c}\text { Mortality } \\
(\%)\end{array}$ & $\begin{array}{c}\text { Animals with } \\
\text { behavioral score }>7 \\
(\%)\end{array}$ \\
\hline $\mathrm{AC}$ & 5 & 0 & 100 \\
$\mathrm{RC}$ & 5 & 0 & 100 \\
$\mathrm{AE}$ & 10 & 10 & 20 \\
$\mathrm{RE}$ & 10 & 20 & 40
\end{tabular}

Animals had a significantly lower behavioral score $(p<0.05)$ after embolism than did control animals. $A C$, Antegrade control; $R C$, retrograde control; $A E$, antegrade embolism; $R E$, retrograde embolism.

Institutes of Health (NIH Publication No. 86-23, revised 1985). The protocol for these experiments was approved by the Mount Sinai Institutional Animal Care and Use Committee.

Animal preparation. Thirty juvenile Yorkshire pigs (Thomas D. Morris, Inc., Reisterstown, Md.), weighing 19 to $28 \mathrm{~kg}$ (mean $22.6 \pm 1.9 \mathrm{~kg}$ ), were used for this study (Table I). Anesthesia was induced with ketamine hydrochloride $(10 \mathrm{mg} / \mathrm{kg}$ intramuscularly) and muscular paralysis maintained with pancuronium $(0.1 \mathrm{mg} / \mathrm{kg}$ intravenously). Endotracheal intubation was performed and the pigs' lungs were ventilated mechanically with air containing more than $40 \%$ oxygen: the minute volume was adjusted to maintain the arterial carbon dioxide tension between 35 and $40 \mathrm{~mm} \mathrm{Hg}$, uncorrected for temperature. Anesthesia was maintained with isoflurane $(1 \%)$.

Catheters were positioned in the left femoral artery and vein for monitoring purposes and withdrawal of blood samples. A thermodilution catheter (93 A $831 \mathrm{H} 7.5 \mathrm{~F}$, Baxter Healthcare Corp, Irvine, Calif.) was inserted into the pulmonary artery to measure cardiac output. By means of a procedure previously described, sagittal sinus cannulation (performed with a 24-gauge intravenous cannula) was carried out to measure cerebral venous pressure and obtain blood gas and metabolic data. Esophageal, rectal, and brain temperatures were measured. Brain temperature was determined with an epidural thermal probe (IT-18, Physitemp Instruments Inc., Clifton, N.J.), which was positioned under the calvarium.

Through a right thoracotomy, the azygos vein was ligated and the superior vena cava (SVC), aortic arch, and descending aorta just distal to the left subclavian artery were mobilized. After heparinization (300 IU $/ \mathrm{kg}$ ), both the ascending aorta and the right atrium were cannulated and cardiopulmonary bypass (CPB) was initiated at a flow rate of $100 \mathrm{ml} / \mathrm{kg}$ per minute. Left ventricular decompression was achieved by cannulation of that structure via a right superior pulmonary vein. $\mathrm{CPB}$ with perfusion cooling was carried out for 30 to 40 minutes to attain an esophageal temperature of $20^{\circ} \mathrm{C}$. At this point cardiac arrest was established by adding potassium chloride $(1 \mathrm{mEq} / \mathrm{kg})$ to the perfusate; it was maintained with topical cardiac cooling. The aorta was crossclamped proximal to the head vessels and, at an esophageal temperature of $20^{\circ} \mathrm{C}$, the descending aorta also was clamped, so that the transverse aortic arch with its arterial. supply to the brain was isolated.

Experimental groups. The animals were initially divided into four experimental groups: an antegrade control group ( $\mathrm{AC}, n=5)$; an antegrade embolism group ( $\mathrm{AE}$, $n=10)$; a retrograde control group $(\mathrm{RC}, n=5)$, and a retrograde embolism group ( $\mathrm{RE}, n=10$ ).

After preliminary analysis of behavioral results, a further 10 animals were subjected to embolism and retrograde perfusion to try to clarify the diverse outcomes seen in the original RE group. Because these retrograde perfusion experiments were conducted after all the controls, the data from the second group of RE animals are excluded from the overall analysis. The animals were used only for comparisons of retrograde cerebral perfusion at different SVC pressures: for these comparisons, this second group was combined with the original RE group to produce the REL (SVC pressure $<40 \mathrm{~mm} \mathrm{Hg}$ ) and $\mathrm{REH}$ (SVC pressure $\geq 40 \mathrm{~mm} \mathrm{Hg}$ ) groups. The experimental groups did not significantly differ in average weights, cooling times, or rewarming times, or in rectal, esophageal, or epidural temperatures at the various time points.

Experimental protocol. In both $\mathrm{RE}$ and $\mathrm{AE}$ groups, $200 \mathrm{mg}$ of polystyrene microspheres $(250$ to $750 \mu \mathrm{m})$ (Duke Scientific Corp., Palo Alto, Calif.) coated with albumin to minimize clustering, suspended in $10 \mathrm{ml}$ saline solution, and vortexed (S8223-1, American Scientific Products, McGaw Park, Ill.) for several minutes were injected into the aortic perfusion cannula, which was positioned in the isolated aortic arch. In the control groups ( $\mathrm{RC}$ and $\mathrm{AC}$ ) $10 \mathrm{ml}$ of saline solution was injected. After bolus injection of the microspheres or saline solution, conventional perfusion was continued for a total of 30 minutes in the antegrade perfusion groups, but for only 5 minutes (long enough to deliver the microspheres) in the retrograde perfusion groups. In all groups perfusion flow was adjusted to maintain the aortic arch pressure at approximately $50 \mathrm{~mm} \mathrm{Hg}$ : aortic pressure was monitored via a pressure line positioned in the isolated aortic arch.

In the retrograde perfusion groups, 25 minutes of flow was initiated in a retrograde fashion via a cannula in the SVC after embolization (or infusion of saline solution). Retrograde perfusion was established gradually, with a flow rate starting at $50 \mathrm{ml} / \mathrm{min}$ and increasing $10 \mathrm{ml} / \mathrm{min}$ every 10 seconds until an adequate amount of flow was achieved. To ensure uniform retrograde cerebral perfusion, we regulated flow in the SVC to produce a pressure of $30 \mathrm{~mm} \mathrm{Hg}$ in the sagittal sinus, regardless of SVC pressure: a separate SVC line was used to monitor perfusion pressure during retrograde perfusion. In the animals undergoing retrograde perfusion, perfusate returning to the isolated aortic arch was collected and the volume recorded; the microspheres were recovered from the perfusate by means of a $40 \mu \mathrm{m}$ transfusion filter.

After completion of either antegrade or retrograde perfusion, the animals were rewarmed, weaned from $\mathrm{CPB}$, and extubated after the last measurement period (4 hours after the start of rewarming).

During the experiments, measurements were recorded at five different times: (1) baseline, at $37^{\circ} \mathrm{C}$ (esophageal); (2) end of cooling, at $20^{\circ} \mathrm{C}$, immediately before institution of intervention; (3) during rewarming, at $30^{\circ} \mathrm{C}$; (4) 2 hours after the start of rewarming; and (5) 4 hours after the start of rewarming.

After the operation, all animals were evaluated daily according to a previously described quantitative behav- 
ioral score that involves the following: mental status $(0=$ comatose or dead, $1=$ stuporous, $2=$ depressed, $3=$ normal); appetite ( $0=$ refuses liquids, $1=$ refuses solids, 2 = decreased, $3=$ normal $)$, and motor function $(0=$ unable to stand, $\mathbb{1}=$ unable to walk, $2=$ unsteady gait, 3 = normal). Numeric summing of these functions provided a final score in which the highest number " 9 " reflects apparently normal neurologic function: a score of 8 means that the animals are able to stand unassisted, and with a score of more than 7 they are likely to recover fully. Lower numbers indicate substantial neurologic damage.

After the fifth postoperative day, all surviving animals were put to death and the brains were harvested. Half of each brain was submitted to the neuropathologist and the other half was completely digested with $10 \mathrm{~N}$ sodium hydroxide to permit recovery of the previously embolized spheres.

Histopathologic analysis. When the animals were put to death, the entire brain was removed and bisected in a midsagittal plane. The left hemisphere of each brain was then immediately fixed by immersion in $4 \%$ formalin. After fixation, the brain was examined grossly. The cerebrum was sectioned in the coronal plane, and the brain stem, cerebellum, and available spinal cord were sectioned in a horizontal plane. Sections were then processed into paraffin, and $5 \mu \mathrm{m}$ sections were stained with hematoxylin and eosin.

Alternate coronal sections of the entire brain of each animal were systematically examined by a single experienced senior neuropathologist (D.W.), blinded both to the experimental design and to the identity and fate of individual animals. He carefully screened each section for the presence or absence of any infarctive or other damage.

Although no formal morphometric analysis of the volume of ischemic injury was carried out, the histologic examination was summarized for each animal by tabulating the number of sections in which infarctive damage was present separately for the neocortex, hippocampus (including the olfactory region), basal ganglia (including the corpus striatum and thalamus), cerebellum, brain stem, and spinal cord: these scores were then added for a total neurohistopathologic score, combining acute and subacute lesions. Hemorrhagic changes and other unusual finding were also noted.

Electroencephalography (EEG). Regional cortical electrical activity was monitored via four $5 \mathrm{~mm}$ epidural recording electrodes (two frontal and two parietal) implanted as previously described. ${ }^{8}$ A reference and ground electrode were implanted anteriorly, over the frontal sinuses. Electrodes were connected to a Spectrum 32 EEG machine (Caldwell Laboratories, Kennewick, Wash.), where the signal was further amplified, digitized, and stored on an optical disk for later analysis. Continuous EEG activity was recorded for 3 minutes at each measurement time point.

Other measurements. Systemic arterial, venous, and sagittal sinus blood samples were obtained to determine $\mathrm{pH}$, oxygen tension, carbon dioxide tension, oxygen saturation, oxygen content, hematocrit value, and hemoglobin value (Ciba-Corning Diagnostic Corp, Medfield, Mass.). If no significant differences in these parameters, or in mean arterial pressure or cardiac output (which were also measured), were present at baseline or at the subsequent measurement time points, these data are not shown. Lactate content was analyzed with a YSI 2300 Stat device (Yellow Springs Instrument Co. Inc., Yellow Springs, Ohio).

Statistical analysis. Quantitative measurements were compared by analysis of variance and $t$ tests or KruskalWallis tests, as appropriate. For variables in which all animals in a group had the same outcome, the data were grouped into two categories, contingency tables were constructed, and $\chi^{2}$ tests were performed. Two-tailed tests were conducted throughout, at a $5 \%$ significance level, except that the Bonferroni correction was used for pairwise comparisons among groups.

\section{Results}

Morbidity and mortality. All animals were in stable condition during the surgical procedure. Three of the 30 animals in the initial study (one in the AE group and two in the RE group) died, and one in the later RE group also died, for an overall mortality of $10 \%$. Three of the four died within the first 24 hours after the operation, and one was put to death on the fourth postoperative day. All had substantial neurologic impairment: they were unable to stand and had poor appetite and abnormal reflexes. At autopsy, two had brain stem hemorrhage, and all but one (in the later RE group) had extremely high histopathologic scores.

The remaining 27 animals survived at least 5 days and behavioral scores were recorded daily. Two of 10 animals in the $\mathrm{AE}$ group and four of 10 animals in the original $\mathrm{RE}$ group recovered with a score above 7 (see Table I). All surviving animals were put to death on the sixth postoperative day, and each brain, including those of the animals that died prematurely, was harvested and studied as previously described.

Behavioral outcome. Neurologic recovery was complete in both the retrograde and antegrade control groups (RC and $\mathrm{AC}$ groups) (Fig. 1).

After embolization, the median behavioral scores after antegrade and retrograde perfusion were lower than those of control animals, documenting that embolization of microspheres had resulted in clinically apparent neurologic injury. The median scores in the embolism groups were not significantly different from one another, however, as shown in Fig. 1, although there are some suggestive differences between antegrade and retrograde embolism groups (AE and RE groups) on the first day after the operation. There was substantial neurologic injury in eight of 10 animals perfused in an antegrade fashion compared with six of 10 in the original retrograde group (behavioral score $\leq 7$ on day 5 ). 


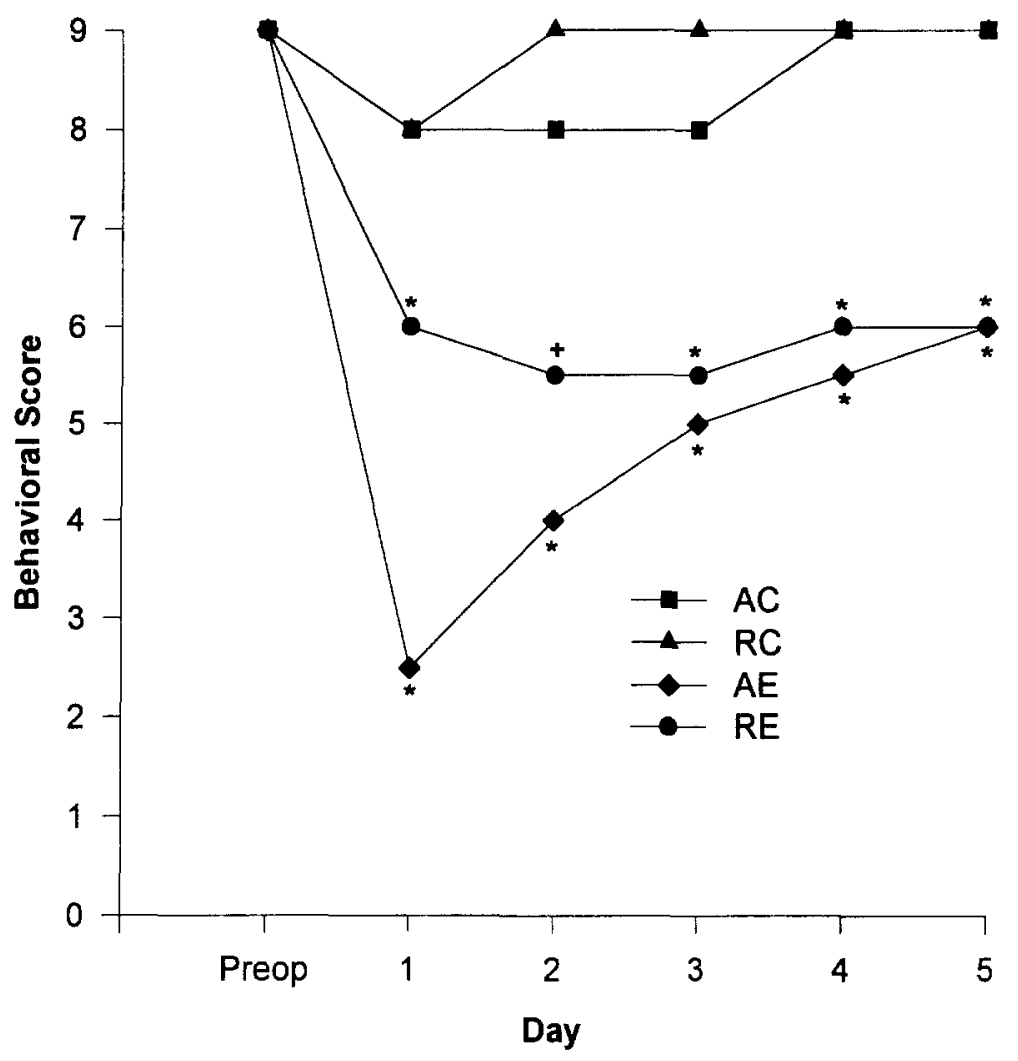

Fig. 1. Median behavioral scores before the operation and for the first 5 days after the operation for the original group of 30 animals. A behavioral score of 9 is normal, a score greater than 7 suggests complete recovery is likely, and a score of 7 or less indicates a significant degree of neurologic impairment, as detailed in the text. Animals that died prematurely were included for the remaining days at their last recorded behavioral score. $A C$, Antegrade control, $n=5 ; R C$, retrograde control, $n=5 ; A E$, antegrade embolism, $n=10 ; R E$, retrograde embolism, $n=10 .{ }^{*} p<0.05$ versus $\mathrm{AC}$ and $\mathrm{RC}$ groups. ${ }^{+} p<0.05$ versus AC group.

In the RE group, however, two animals had no apparent neurologic injury and two had only minimal deficits, suggesting that retrograde cerebral perfusion may mitigate the adverse effect of arterial emboli in at least some cases. On further examination, it became apparent that those animals in the RE group that had a good neurologic outcome (behavioral score $>7$ ) were perfused with lower SVC pressures $(<40 \mathrm{~mm} \mathrm{Hg}$ ) than the animals that had severe neurologic injury.

To further investigate the apparent association between a low SVC pressure during RCP after embolization and a more favorable behavioral outcome, we conducted 10 additional RE experiments. Fig. 2 shows the relatively high behavioral scores beginning on day 1 in the REL group (SVC pressure $<40 \mathrm{~mm} \mathrm{Hg}$ ).
Fig. 3 shows the relationship between SVC pressure and behavioral outcome (in the original retrograde perfusion group) that prompted investigation of the additional animals and enabled subsequent division of the entire retrograde perfusion group into REL and REH subgroups. When the two SVC pressure subgroups were compared, no differences in parameters including perfusion flows, aortic arch return, temperatures, blood gases, metabolic data, or EEG recovery were found that would correlate and perhaps shed light on the striking difference in behavioral outcome between the REL and REH groups.

Histopathologic results. The results of histopathologic examination correlate reasonably well with behavioral evaluations. The histopathologic findings for each animal are detailed in Tables II 


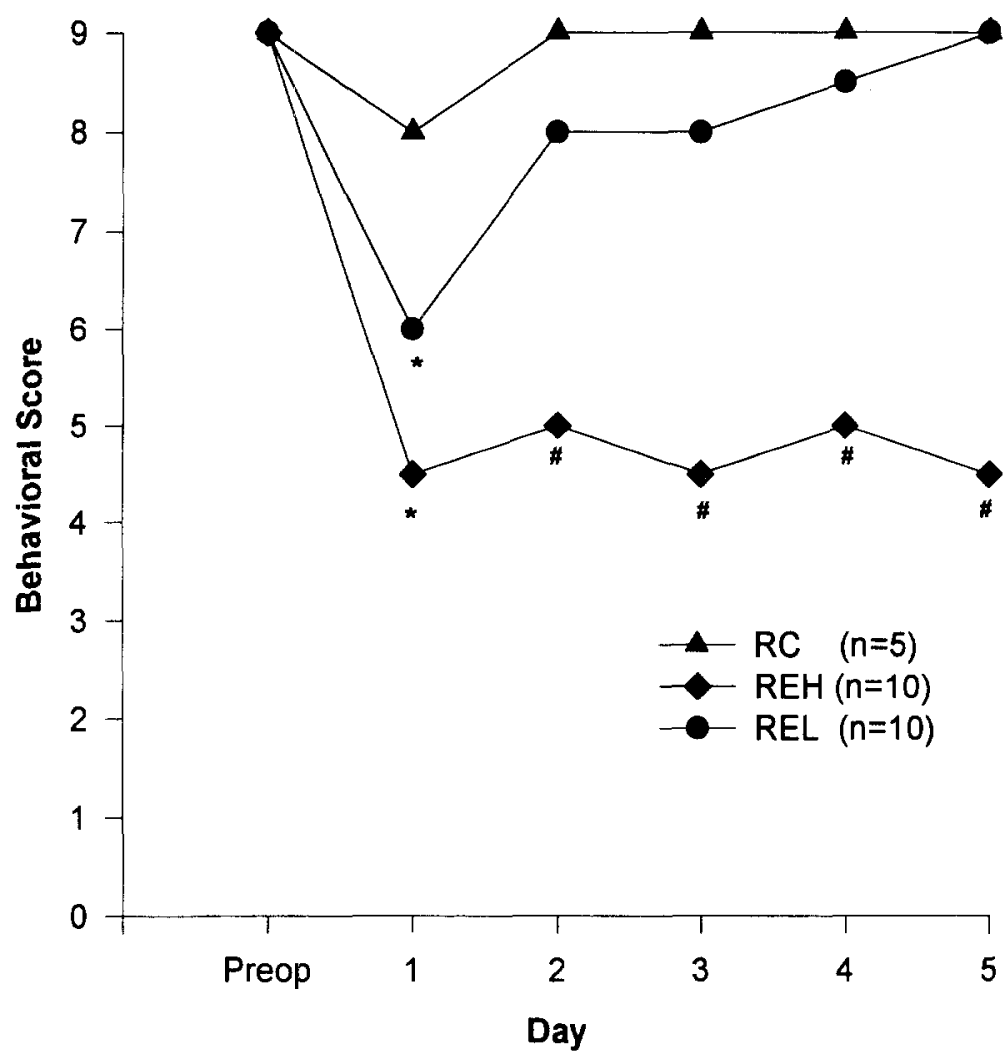

Fig. 2. Median behavioral scores before the operation and for the first 5 days after the operation in all animals that underwent retrograde cerebral perfusion. Animals in the REL group were perfused at low SVC pressures after embolization $(<40 \mathrm{~mm} \mathrm{Hg}$ ), whereas animals in the REH group required higher SVC pressures ( $\geq 40 \mathrm{~mm} \mathrm{Hg}$ ) to obtain adequate sagittal sinus pressures during RCP after embolization. Description of behavioral scores and abbreviations as in Fig. $1 .{ }^{*} p<0.05$ versus RC group. $\# p<0.05$ versus RC and REL groups.

and III, and the median total histopathologic scores are shown in Fig. 4.

Fig. 5 shows the negative relationship between total histopathologic score and behavioral score on day 5 in the original 30 animals: the point for a histopathologic score of zero and a behavioral score of nine represents eight animals. Because these data are not suitable for statistical regression analysis, we have grouped the data as shown in Table IV, which shows a significant relationship $(p<0.02)$ between histopathologic and behavioral scores: a high behavioral score $(>7)$, indicating good neurologic recovery, is usually associated with a low histopathologic score $(\leq 7)$, and a high histopathologic score $(>7)$ is generally found in animals with a behavioral score indicating neurologic impairment $(\leq 7)$.

There were absolutely no abnormalities in the brains of any of the $\mathrm{AC}$ animals, correlating with essentially normal behavioral scores. Among the RC animals, however, despite very good behavioral scores, there were some ischemic lesions, principally in the neocortex, in two animals: these lesions did not invariably correlate with high SVC pressures.

In contrast to the control groups, all but one animal exhibited at least a single area of ischemic injury on histopathologic examination after arterial embolization followed by antegrade perfusion (AE group). This result correllates well with the depressed behavioral scores on the first postoperative day in this group, without marked improvement by the fifth day. The histopathologic evidence indicates again that injection of arterial emboli followed by continued antegrade perfusion does cause brain injury: the median histopathology score was significantly higher in the $\mathrm{AE}$ group than in the control groups $(p<0.01)$ (see Fig. 4).

In the RE group, the results are somewhat more complicated. The initial RE group showed signifi- 


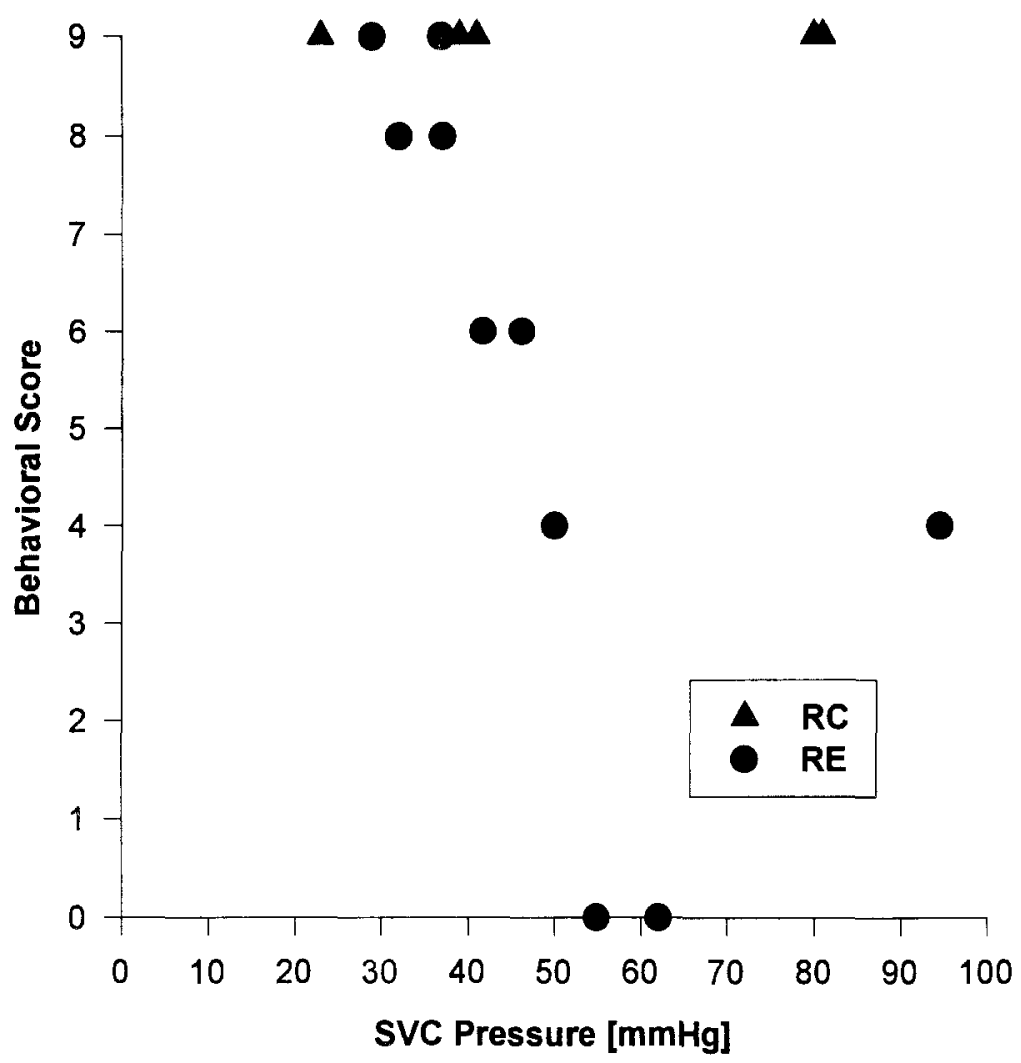

Fig. 3. The relationship of behavioral score on day 5 to SVC pressure during retrograde cerebral perfusion in the first 15 animals subjected to retrograde perfusion. Although behavioral score in the control group (RC) was uniformly high regardless of SVC perfusion pressure, behavioral score in the retrograde embolism (RE) group appeared to be better in those animals perfused at lower SVC pressures. The results in the additional 10 animals subsequently perfused in a retrograde fashion confirmed this original observation.

cant behavioral impairment on the first postoperative day, although less severe than in the AE group, with no significant improvement by day 5 . However, despite slightly better behavioral outcome than in the $\mathrm{AE}$ group, histopathologic damage in the $\mathrm{RE}$ animals was the most severe seen in any of the original groups, with the median values suggesting a failure of retrograde perfusion to protect the brain from embolic injury. Close inspection, however, shows that the histopathologic results, like the behavioral scores, are again clustered at two extremes: several animals with very high scores, and a few with little or no histologic damage.

When the additional 10 animals later subjected to embolism and retrograde perfusion are included in the analysis, and the animals requiring high SVC pressure for adequate retrograde perfusion after embolization (REH) are separated from those requiring less than $40 \mathrm{~mm} \mathrm{Hg}$ (REL), both the behav- ioral scores and the histopathology scores indicate a better outcome after retrograde perfusion at low pressure (REL) than after perfusion at high pressure (REH) (see Figs. 2 and 4). The results in the REL group are not significantly worse than those of the RC group and appear to be better than after antegrade perfusion following embolization (AE).

In contrast, the animals requiring high SVC pressures for retrograde perfusion after embolization (REH) have behavioral scores comparable with those treated with antegrade perfusion $(\mathrm{AE})$, and a high incidence of histopathologic lesions, significantly higher than either the REL group or the control groups. Of the animals undergoing retrograde cerebral perfusion at low pressure after embolization (REL), four of 10 had no histopathologic abnormalities, in contrast to only one of 10 in the $\mathrm{AE}$ group and none in the REH group. 
Volume 110, Number 5

Table II. Histopathologic analysis

\begin{tabular}{|c|c|c|c|c|c|c|c|c|c|c|}
\hline \multirow[b]{2}{*}{ Group } & \multirow[b]{2}{*}{ Neocortex } & \multirow[b]{2}{*}{ Hippocampus } & \multirow{2}{*}{$\begin{array}{c}\text { Basal } \\
\text { ganglia }\end{array}$} & \multirow[b]{2}{*}{ Cerebellum } & \multirow{2}{*}{$\begin{array}{l}\text { Brain } \\
\text { stem }\end{array}$} & \multirow{2}{*}{$\begin{array}{c}\text { Spinal } \\
\text { cord }\end{array}$} & \multirow{2}{*}{$\begin{array}{c}\text { Total } \\
\text { pathology } \\
\text { scores }\end{array}$} & \multicolumn{2}{|c|}{ Behavioral score } & \multirow{2}{*}{$\begin{array}{c}S V C \\
\text { pressure } \\
(\mathrm{mm} H g)\end{array}$} \\
\hline & & & & & & & & Day 1 & Day 5 & \\
\hline $\mathrm{AC}$ & 0 & 0 & 0 & 0 & 0 & 0 & 0 & 8 & 9 & \\
\hline $\mathrm{AC}$ & 0 & 0 & 0 & 0 & 0 & 0 & 0 & 8 & 9 & \\
\hline $\mathrm{AC}$ & 0 & 0 & 0 & 0 & 0 & 0 & 0 & 8 & 9 & \\
\hline $\mathrm{AC}$ & 0 & 0 & 0 & 0 & 0 & 0 & 0 & 8 & 8 & \\
\hline $\mathrm{AC}$ & 0 & 0 & 0 & 0 & 0 & 0 & 0 & 8 & 9 & \\
\hline Median & 0.0 & 0.0 & 0.0 & 0.0 & 0.0 & 0.0 & 0.0 & 8.0 & 9.0 & \\
\hline $\mathrm{RC}$ & 5 & 0 & 1 & 0 & 0 & 0 & 6 & 7 & 9 & 80 \\
\hline $\mathrm{RC}$ & 1 & 0 & 0 & 0 & 0 & 0 & 1 & 8 & 9 & 23 \\
\hline $\mathrm{RC}$ & 0 & 0 & 0 & 0 & 0 & 0 & 0 & 8 & 9 & 80 \\
\hline $\mathrm{RC}$ & 0 & 0 & 0 & 0 & 0 & 0 & 0 & 8 & 9 & 39 \\
\hline $\mathrm{RC}$ & 0 & 0 & 0 & 0 & 0 & 0 & 0 & 8 & 9 & 41 \\
\hline Median & 0.0 & 0.0 & 0.0 & 0.0 & 0.0 & 0.0 & 0.0 & 8.0 & 9.0 & $53 \pm 26$ \\
\hline $\mathrm{AE}$ & 6 & 3 & 0 & 0 & 0 & 0 & 9 & 3 & 6 & \\
\hline $\mathrm{AE}$ & 10 & 2 & 1 & 0 & 0 & 0 & 13 & 0 & 0 & \\
\hline $\mathrm{AE}$ & 0 & 1 & 1 & 1 & 0 & 0 & 3 & 3 & 5 & \\
\hline $\mathrm{AE}$ & 0 & 0 & 0 & 1 & 0 & 0 & 1 & 5 & 6 & \\
\hline $\mathrm{AE}$ & 0 & 1 & 2 & 2 & 0 & 0 & 5 & 4 & 8 & \\
\hline $\mathrm{AE}$ & 0 & 0 & 0 & 1 & 0 & 0 & 1 & 4 & 6 & \\
\hline $\mathrm{AE}$ & 3 & 1 & 4 & 2 & 0 & 0 & 10 & 2 & 1 & \\
\hline $\mathrm{AE}$ & 0 & 0 & 0 & 0 & 0 & 0 & 0 & 2 & 8 & \\
\hline $\mathrm{AE}$ & 2 & 1 & 0 & 1 & 0 & 0 & 4 & 2 & 6 & \\
\hline $\mathrm{AE}$ & 0 & 0 & 2 & 2 & 0 & 0 & 4 & 2 & 7 & \\
\hline Median & 0.0 & 1.0 & 0.5 & 1.0 & 0.0 & 0.0 & 4.0 & 2.5 & 6.0 & \\
\hline $\mathrm{RE}$ & 1 & 0 & 0 & 3 & 0 & 0 & 4 & 5 & 4 & 50 \\
\hline RE & 3 & 2 & 2 & 2 & 0 & 0 & 9 & 6 & 8 & 32 \\
\hline RE & 9 & 3 & 0 & 3 & 1 & 0 & 16 & 6 & 0 & 62 \\
\hline $\mathrm{RE}$ & 0 & 0 & 0 & 0 & 0 & 0 & 0 & 8 & 9 & 29 \\
\hline $\mathrm{RE}$ & 9 & 0 & 0 & 4 & 0 & 1 & 14 & 5 & 6 & 46 \\
\hline $\mathrm{RE}$ & 0 & 1 & 2 & 0 & 0 & 0 & 3 & 6 & 9 & 37 \\
\hline $\mathrm{RE}$ & 6 & 3 & 2 & 1 & 0 & 0 & 12 & 4 & 4 & 95 \\
\hline RE & 6 & 0 & 2 & 2 & 1 & 0 & 11 & 6 & 6 & 42 \\
\hline $\mathrm{RE}$ & 10 & 1 & 2 & 3 & 0 & 0 & 16 & 6 & 8 & 37 \\
\hline RE & 9 & 1 & 3 & 0 & 0 & 0 & 13 & 0 & 0 & 55 \\
\hline Median & 6.0 & 1.0 & 2.0 & 2.0 & 0.0 & 0.0 & 11.5 & 6.0 & 6.0 & $48 \pm 19$ \\
\hline
\end{tabular}

Histopathologic and behavioral scores are medians; SVC pressures are mean \pm standard deviation. Hippocampus includes olfactory and basal ganglia includes thalamus. $A C$, Antegrade control; $R C$, retrograde control; $A E$, antegrade embolism; $R E$, retrograde embolism.

EEG results. No difference in total EEG power between groups was present at baseline or at the end of cooling: the $\mathrm{EEG}$ was isoelectric at $20^{\circ} \mathrm{C}$ in all the animals. At $30^{\circ} \mathrm{C}$ during rewarming, the animals in the antegrade groups ( $\mathrm{AC}$ and $\mathrm{AE}$ ) had earlier EEG return than those in the retrograde groups ( $\mathrm{RC}$ and $\mathrm{RE}, p<0.05$ ) (Fig. 6). This significant difference was sustained at the measurements 2 and 4 hours after the start of rewarming. EEG recovery was better in control animals (AC and RC) than in the embolism animals (AE and RE) at 2 hours ( $p<$ 0.05) (Fig. 6). At this time point, the animals showed significantly slower recovery after embolism and retrograde perfusion (RE group) than after embolism followed by antegrade perfusion (AE group).
Microsphere results. After digestion of the brains after the animals were put to death, no significant difference in microsphere recovery was found among the various embolism groups, although the trend was in the direction one might predict, with slightly fewer residual emboli in the brains after retrograde perfusion (RE group). Recovery from the initial RE group averaged slightly lower, $1.10 \pm 0.78 \mathrm{mg}$, than recovery from the $\mathrm{AE}$ group, $1.14 \pm 0.24 \mathrm{mg}$.

Adding the new group of RE animals and dividing them into the two SVC pressure subgroups yielded somewhat fewer microspheres recovered in the REL (low SVC pressure) group $(0.6 \pm 0.6 \mathrm{mg})$ than in the high SVC pressure group, REH $(0.9 \pm 0.7$ 
Table III. Histopathologic analysis

\begin{tabular}{|c|c|c|c|c|c|c|c|c|c|c|}
\hline \multirow[b]{2}{*}{ Group } & \multirow[b]{2}{*}{ Neocortex } & \multirow[b]{2}{*}{ Hippocampus } & \multirow{2}{*}{$\begin{array}{c}\text { Basal } \\
\text { ganglia }\end{array}$} & \multirow[b]{2}{*}{ Cerebellum } & \multirow{2}{*}{$\begin{array}{l}\text { Brain } \\
\text { stem }\end{array}$} & \multirow{2}{*}{$\begin{array}{c}\text { Spinal } \\
\text { cord }\end{array}$} & \multirow{2}{*}{$\begin{array}{c}\text { Total } \\
\text { pathology } \\
\text { scores }\end{array}$} & \multicolumn{2}{|c|}{ Behavioral score } & \multirow{2}{*}{$\begin{array}{c}S V C \\
\text { pressure } \\
\text { (mm Hg) }\end{array}$} \\
\hline & & & & & & & & Day 1 & Day 5 & \\
\hline $\mathrm{RC}$ & 5 & 0 & 1 & 0 & 0 & 0 & 6 & 7 & 9 & 80 \\
\hline $\mathrm{RC}$ & 1 & 0 & 0 & 0 & 0 & 0 & 1 & 8 & 9 & 23 \\
\hline $\mathrm{RC}$ & 0 & 0 & 0 & 0 & 0 & 0 & 0 & 8 & 9 & 80 \\
\hline $\mathrm{RC}$ & 0 & 0 & 0 & 0 & 0 & 0 & 0 & 8 & 9 & 39 \\
\hline $\mathrm{RC}$ & 0 & 0 & 0 & 0 & 0 & 0 & 0 & 8 & 9 & 41 \\
\hline Median & 0.0 & 0.0 & 0.0 & 0.0 & 0.0 & 0.0 & 0.0 & 8.0 & 9.0 & $53 \pm 26$ \\
\hline REL & 3 & 2 & 2 & 2 & 0 & 0 & 9 & 6 & 8 & 32 \\
\hline REL & 0 & 0 & 0 & 0 & 0 & 0 & 0 & 8 & 9 & 29 \\
\hline REL & 0 & 1 & 2 & 0 & 0 & 0 & 3 & 6 & 9 & 37 \\
\hline REL & 10 & 1 & 2 & 3 & 0 & 0 & 16 & 6 & 8 & 37 \\
\hline REL & 0 & 0 & 0 & 0 & 0 & 0 & 0 & 5 & 9 & 38 \\
\hline REL & 0 & 0 & 0 & 1 & 0 & 0 & 1 & 6 & 9 & 36 \\
\hline REL & 0 & 0 & 0 & 4 & 0 & 0 & 4 & 7 & 9 & 35 \\
\hline REL & 0 & 0 & 0 & 0 & 0 & 0 & 0 & 6 & 9 & 33 \\
\hline REL & 0 & 0 & 0 & 0 & 0 & 0 & 0 & 8 & 9 & 36 \\
\hline REL & 0 & 0 & 0 & 1 & 0 & 0 & 1 & 5 & 8 & 35 \\
\hline Median & 0.0 & 0.0 & 0.0 & 0.5 & 0.0 & 0.0 & 1.0 & 6.0 & 9.0 & $35 \pm 3$ \\
\hline REH & 1 & 0 & 0 & 3 & 0 & 0 & 4 & 5 & 4 & 50 \\
\hline REH & 9 & 3 & 0 & 3 & 1 & 0 & 16 & 6 & 0 & 62 \\
\hline REH & 9 & 0 & 0 & 4 & 0 & 1 & 14 & 5 & 6 & 46 \\
\hline REH & 6 & 3 & 2 & 1 & 0 & 0 & 12 & 4 & 4 & 95 \\
\hline REH & 6 & 0 & 2 & 2 & 1 & 0 & 11 & 6 & 6 & 42 \\
\hline REH & 9 & 1 & 3 & 0 & 0 & 0 & 13 & 0 & 0 & 55 \\
\hline REH & 0 & 0 & 0 & 2 & 0 & 0 & 2 & 4 & 6 & 52 \\
\hline REH & 4 & 1 & 0 & 2 & 0 & 0 & 7 & 2 & 5 & 107 \\
\hline REH & 0 & 0 & 0 & 2 & 1 & 0 & 3 & 4 & 0 & 84 \\
\hline REH & 0 & 0 & 0 & 3 & 0 & 0 & 3 & 5 & 7 & 84 \\
\hline Median & 5.0 & 0.0 & 0.0 & 2.0 & 0.0 & 0.0 & 9.0 & 4.5 & 4.5 & $68 \pm 23$ \\
\hline
\end{tabular}

Histopathologic and behavioral scores are medians; SVC pressures are mean \pm standard deviation. Hippocampus includes olfactory and basal ganglia includes thalamus. $R C$, Retrograde control; $R E L, \mathrm{RE}$ with low SVC pressure; $R E H$, RE with high SVC pressure.

$\mathrm{mg}$ ), although these differences were not statistically significant.

Aortic arch blood return during retrograde perfusion. In both RC and RE groups, the volume of blood recovered from the isolated arch was low $(0.80 \pm 0.35 \mathrm{ml} / \mathrm{kg}$ per minute in the $\mathrm{RC}$ group compared with $0.46 \pm 0.15 \mathrm{ml} / \mathrm{kg}$ per minute in the RE group) and not statistically different. This represents a return of $3.7 \% \pm 2$ of total retrograde flow in the $\mathrm{RC}$ group and $3.4 \% \pm 2$ in the $\mathrm{RE}$ group (Table V).

Comparability of groups: Hemodynamic and blood gas data. CPB data and animal weights were comparable in all groups: there were no significant differences in the CPB times between groups during either cooling or rewarming. With cooling to an esophageal temperature of $20^{\circ} \mathrm{C}$, epidural temperature tended to keep pace with esophageal temperature, and rectal temperature lagged behind, with no significant differences between experimental groups.
Hemodynamic and blood gas data show that all animals were in stable condition before, during, and after bypass and that no significant differences among groups in either blood gas or hematocrit results were noted during the interventions. The late $R E$ group also showed no significant differences from the other groups.

Pump flow and vascular resistance. Perfusion flow rate in the isolated aortic arch (with the aortic arch pressure maintained at approximately $50 \mathrm{~mm}$ $\mathrm{Hg}$ during antegrade flow) was between $13.3 \pm 5.6$ and $24.4 \pm 12.3 \mathrm{ml} / \mathrm{kg}$ per minute immediately before injection of either the spheres or saline solution. There was a statistically significant difference between the marked decrease in flow that occurred in the embolized groups compared with the control groups, in which a very slight flow decrement after injection of saline solution was noted.

Vascular resistance changes were calculated from these data. Vascular resistance increased signifi- 


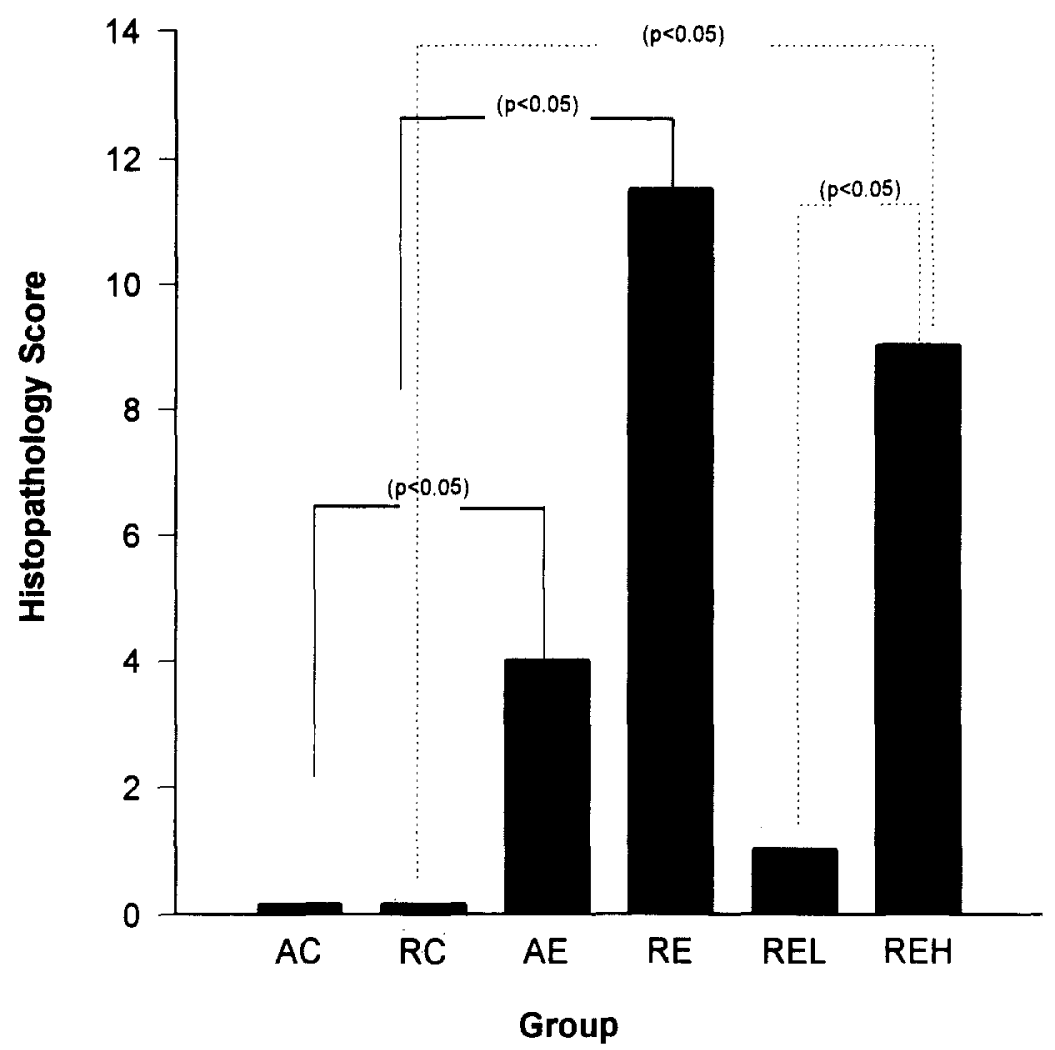

Fig. 4. Median total histopathology scores for the four original experimental groups and all the RE animals after dividing into the two pressure subgroups (REL and REH): $A C$, antegrade control, $n=5 ; R C$, retrograde control, $n=5 ; A E$, antegrade embolism, $n=10 ; R E$, original retrograde embolism group; $n=$ $10, R E L$, retrograde perfusion at low SVC pressure $(n=10) ; R E H$, retrograde perfusion at high SVC pressure $(n=10)$ (as in Fig. 2). The total histopathology score for each animal was the sum of all sections from the neocortex, hippocampus, cerebellum, brain stem, and spinal cord that showed infarctive damage: a higher score therefore indicates a worse outcome. Data are from Tables II and III. Each embolism group differed significantly from its corresponding control ( $\mathrm{RE}$ versus $\mathrm{RC}, p<0.05$; $\mathrm{AE}$ versus $\mathrm{AC}, p<0.01$ by a $\chi^{2}$ test comparing the proportion of animals that were free of damage, as indicated by a histopathologic score of zero).

cantly after microspheres were injected, whereas there were minimal alterations of vascular resistance in the control groups: the significantly higher vascular resistance seen in the embolized groups $(0.42 \pm$ 0.2 versus $0.20 \pm 0.1 \mathrm{~mm} \mathrm{Hg} / \mathrm{ml}$ per minute) documents the effectiveness of the embolization.

During the subsequent 25 minutes of antegrade perfusion, the flow rate was $11.0 \pm 3.1 \mathrm{ml} / \mathrm{kg}$ per minute in the $\mathrm{AC}$ group and $7.2 \pm 3.8 \mathrm{ml} / \mathrm{kg}$ per minute in AE group. Similarly, during the 25 minutes of retrograde perfusion, the flow rate was $34.6 \pm 32 \mathrm{ml} / \mathrm{kg}$ per minute in the $\mathrm{RC}$ group and $17.5 \pm 10 \mathrm{ml} / \mathrm{kg}$ per minute in the RE group (see Table V).
Pressure measurements. Aortic arch pressure was maintained at 50 to $60 \mathrm{~mm} \mathrm{Hg}$ in both antegrade groups during the antegrade perfusion period.

Sagittal sinus pressure was maintained at $30 \mathrm{~mm}$ $\mathrm{Hg}$ in the $\mathrm{RE}$ and $\mathrm{RC}$ groups during retrograde perfusion, except in one animal in the RE group. In this single pig, it was not possible to maintain a sagittal sinus pressure of $30 \mathrm{~mm} \mathrm{Hg}$ despite SVC pressure in excess of $90 \mathrm{~mm} \mathrm{Hg}$; thus this animal was perfused with a sagittal sinus pressure of only $19 \mathrm{~mm}$ $\mathrm{Hg}$ despite a high SVC pressure $(95 \mathrm{~mm} \mathrm{Hg})$. During retrograde cerebral perfusion, SVC pressures in the remaining animals varied between 23 and $107 \mathrm{~mm} \mathrm{Hg}$ and are shown in Tables II and III. 


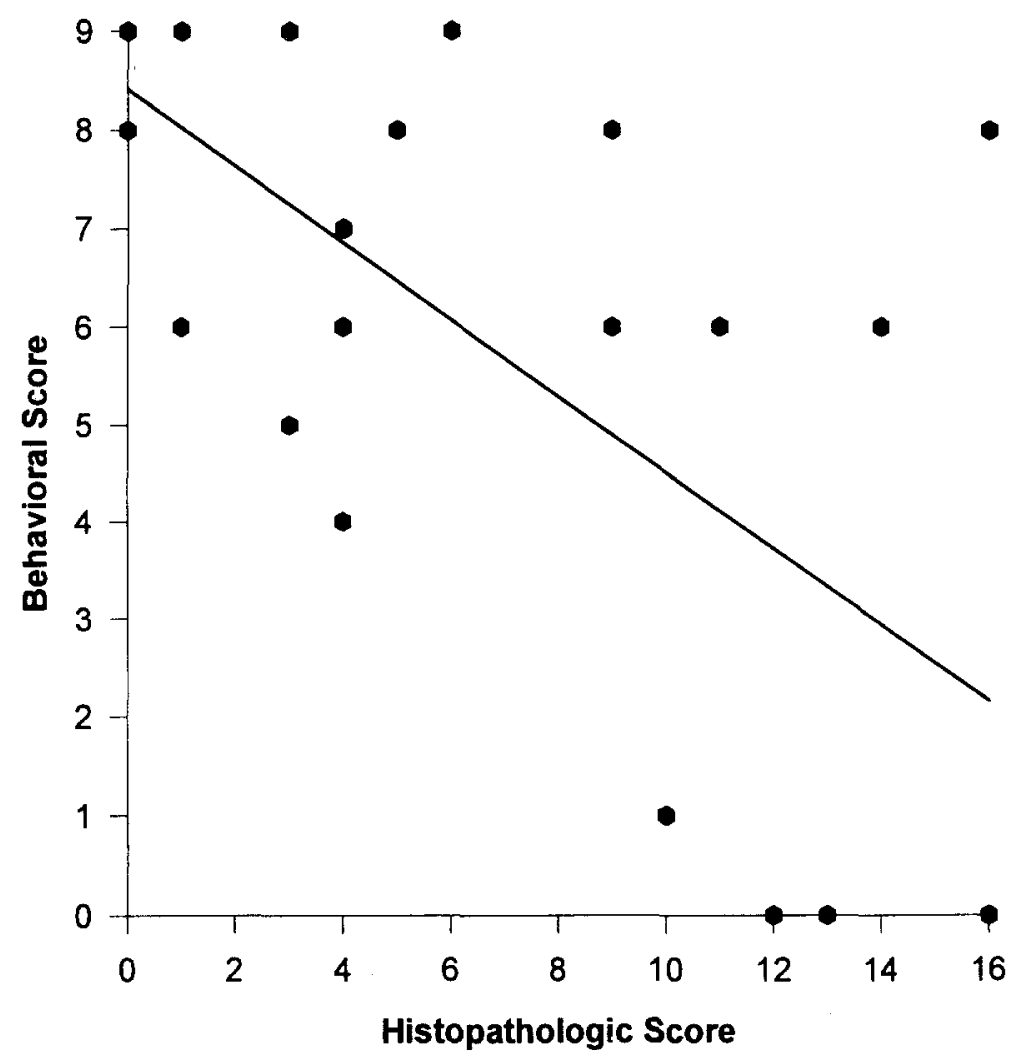

Fig. 5. The negative relationship between behavioral score on day 5 and histopathologic score is shown for the original 30 animals in the protocol. As discussed in the text, these data are not suitable for a statistical regression analysis: the strength of this negative correlation is shown by grouping the data as shown in Table IV.

Table IV. Histopathology score

\begin{tabular}{lc}
\hline Behavioral score $>7$ & Histopathology score \\
\hline$n=14(70 \%)$ & $<7(n=20)$ \\
$n=2(20 \%)$ & $>7(n=10)$ \\
\hline
\end{tabular}

A highly significant relationship $(p<0.02)$ exists between histopathology and behavioral scores: a high behavioral score $(>7)$ is usually associated with a low histopathology score $(<7)$.

Metabolic data. Cerebral oxygen extraction and sagittal sinus lactate measurements (Table VI), behaved as expected at $20^{\circ} \mathrm{C}$ : cerebral oxygen extraction decreased to $42 \% \pm 5 \%$ of baseline and sagittal sinus lactate increased $73 \% \pm 5 \%$ in all groups.

On rewarming $\left(30^{\circ} \mathrm{C}\right)$, cerebral oxygen extraction and sagittal sinus lactate concentration increased in all groups, although oxygen extraction was statistically lower and lactate concentration was significantly higher in both retrograde perfusion groups when compared with the antegrade groups. Two hours after the start of rewarming, all groups except the RE group showed recovery to near baseline values: at 4 hours, the metabolic determinants in all groups without exception had returned to normal. Without concomitant measurements of cerebral blood flow, the significance of these observations is somewhat problematic, but the results suggest that retrograde perfusion did not support ongoing metabolism as fully as antegrade perfusion and that the oxygen debt was most severe in the RE group.

\section{Discussion}

The theoretic appeal of hypothermic retrograde cerebral perfusion as a way of improving cerebral protection during cardiac surgery-by potentially mitigating both ischemic and embolic injury--has led to the fairly widespread clinical use of retrograde perfusion despite the fact that little is really known about either its efficacy or its safety. ${ }^{9-14}$ A previous study from our laboratory suggests that retrograde perfusion during 90 minutes of hypothermic circulatory arrest at $20^{\circ} \mathrm{C}$ does provide some nutritive flow, helps maintain low cerebral temperatures during prolonged hypothermia, and improves both be- 


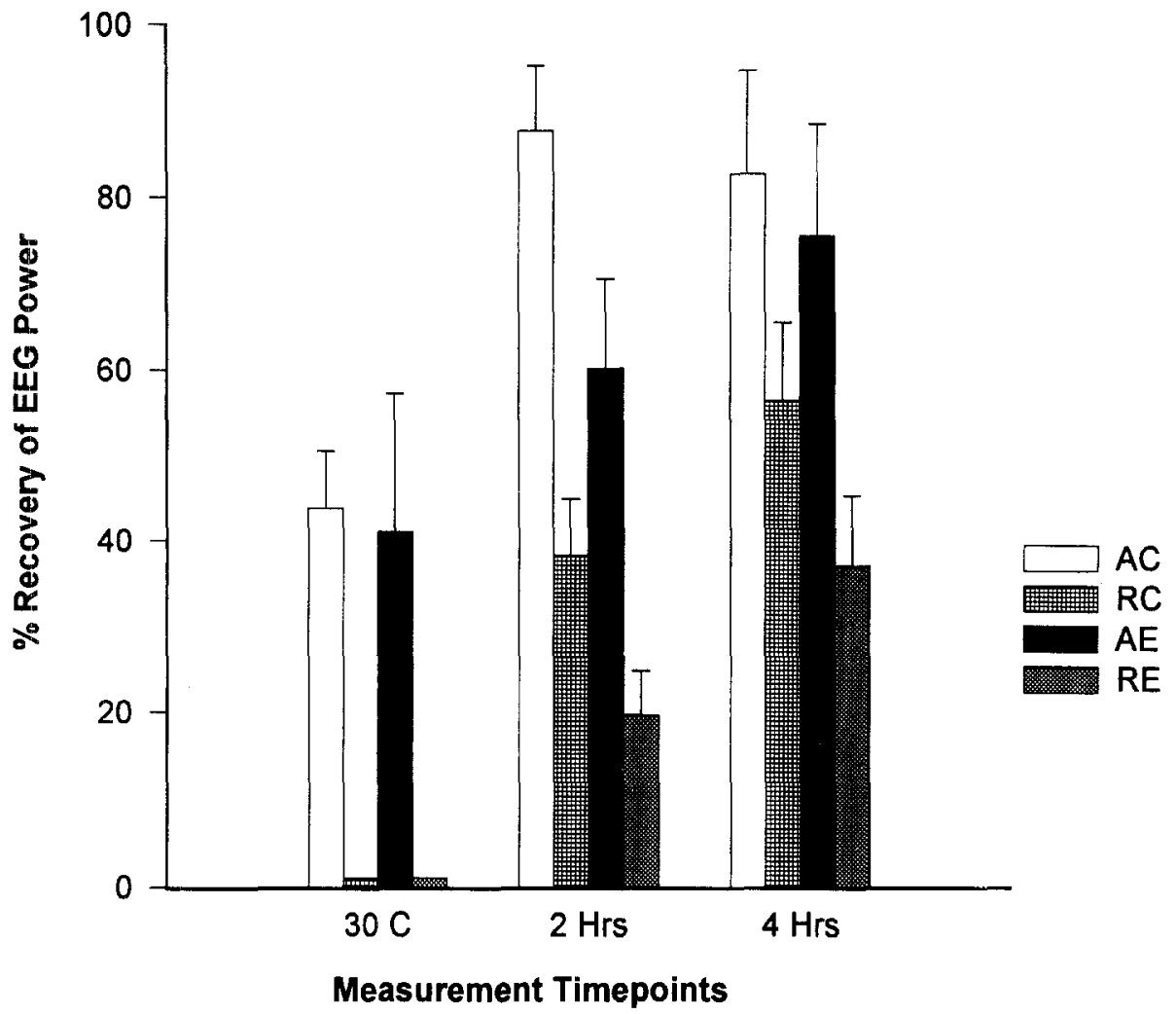

Fig. 6. Percent recovery of total EEG power during rewarming, at 2 and 4 hours after embolization following antegrade or retrograde perfusion, and also in controls (mean \pm standard error). Data were obtained during 3-minute intervals of EEG recording from two frontal and two parietal electrodes. Groups and abbreviations are as in Fig. 1. Recovery after antegrade perfusion was more rapid than after retrograde perfusion, with significantly greater total power in all animals after antegrade perfusion than after retrograde perfusion at all time points. At 2 hours, however, there was also a significant difference between animals that had undergone embolization compared with the appropriate control animals $(p<0.05$ for $\mathrm{AC}$ versus $\mathrm{AE}$ and also for $\mathrm{RC}$ versus $\mathrm{RE}$ ), correlating with the differences in behavioral outcomes in embolism and control animals.

Table V. Antegrade/retrograde CPB flow, SVC pressure, and aortic arch return

\begin{tabular}{|c|c|c|c|c|c|}
\hline \multirow[b]{2}{*}{ Group } & \multicolumn{2}{|c|}{$C P B$ flow } & \multirow[b]{2}{*}{$\begin{array}{c}\text { SVC pressure } \\
(\mathrm{mm} \mathrm{Hg})\end{array}$} & \multicolumn{2}{|c|}{ Aortic arch blood retum } \\
\hline & $\begin{array}{l}\text { Antegrade } \\
(\mathrm{ml} / \mathrm{kg} / \mathrm{min})\end{array}$ & $\begin{array}{l}\text { Retrograde } \\
(\mathrm{ml} / \mathrm{kg} / \mathrm{min})\end{array}$ & & $\begin{array}{l}\text { Volume } \\
(\mathrm{ml} / \mathrm{kg} / \mathrm{min})\end{array}$ & \%o of flow rate \\
\hline $\mathrm{AC}$ & $11.0 \pm 3.1$ & & & & \\
\hline $\mathrm{RC}$ & & $34.6 \pm 32.7$ & $53 \pm 26$ & $0.8 \pm 0.4$ & $3.7 \pm 1.9$ \\
\hline $\mathrm{AE}$ & $7.2 \pm 3.8$ & & & & \\
\hline RE & & $17.5 \pm 10.3$ & $48 \pm 19$ & $0.5 \pm 0.2$ & $3.4 \pm 1.9$ \\
\hline
\end{tabular}

CPB flow was significantly lower $(p<0.05)$ in the antegrade embolism group than in the controls. Values are shown as mean \pm standard deviation. $A C$, antegrade conkrol; $R C$, retrograde control; $A E$, antegrade embolism; $R E$, retrograde embolism.

havioral and histopathologic outcome when compared with hypothermia alone, even when the head is packed in ice. ${ }^{15}$

The current study was an attempt in our chronic animal model to ascertain the possible efficacy of retrograde cerebral perfusion in protecting against cerebral injury from emboli released into the ascending aorta during cardiac or aortic operations. The evidence we have obtained in this preliminary investigation suggests that retrograde perfusion may be a promising tool for limiting brain injury from acute aortic emboli in some individuals. 
Table VI. Metabolic data

\begin{tabular}{|c|c|c|c|c|c|}
\hline & $\begin{array}{c}\text { Baseline } \\
\left(37^{\circ} \mathrm{C}\right)\end{array}$ & $\begin{array}{l}\text { End of cooling } \\
\qquad\left(20^{\circ} \mathrm{C}\right)\end{array}$ & $\begin{array}{c}\text { Rewarming } \\
\left(30^{\circ} \mathrm{C}\right)\end{array}$ & $\begin{array}{c}2 \mathrm{hr} \text { after } \\
\text { intervention }\end{array}$ & $\begin{array}{l}4 \mathrm{hr} \text { after } \\
\text { intervention }\end{array}$ \\
\hline \multicolumn{6}{|c|}{ Cerebral oxygen extraction $(\mathrm{ml} / \mathrm{dl})$} \\
\hline $\mathrm{AC}$ & $4.2 \pm 0.6$ & $2.5 \pm 1.3$ & $6.2 \pm 1.1$ & $4.6 \pm 0.7$ & $5.3 \pm 1.0$ \\
\hline $\mathrm{RC}$ & $4.1 \pm 2.7$ & $2.4 \pm 0.7$ & $1.7 \pm 1.5$ & $5.6 \pm 1.6$ & $6.0 \pm 1.5$ \\
\hline $\mathrm{AE}$ & $3.9 \pm 1.6$ & $2.0 \pm 1.4$ & $4.1 \pm 1.7$ & $5.2 \pm 1.6$ & $5.1 \pm 1.6$ \\
\hline $\mathrm{RE}$ & $4.8 \pm 1.3$ & $1.5 \pm 0.5$ & $2.6 \pm 1.4$ & $6.2 \pm 1.5$ & $5.8 \pm 1.5$ \\
\hline \multicolumn{6}{|c|}{ Sagittal sinus lactate (mmol/L) } \\
\hline $\mathrm{AC}$ & $1.2 \pm 0.6$ & $2.2 \pm 1.3$ & $3.7 \pm 1.7$ & $3.9 \pm 1.0$ & $2.0 \pm 0.6$ \\
\hline $\mathrm{RC}$ & $1.2 \pm 0.5$ & $2.3 \pm 0.8$ & $5.5 \pm 1.5$ & $4.6 \pm 1.6$ & $2.2 \pm 0.9$ \\
\hline $\mathrm{AE}$ & $1.2 \pm 0.5$ & $1.8 \pm 0.5$ & $3.6 \pm 1.8$ & $3.4 \pm 1.4$ & $2.0 \pm 0.9$ \\
\hline RE & $1.4 \pm 0.6$ & $2.1 \pm 0.6$ & $5.1 \pm 2.1$ & $4.2 \pm 1.8$ & $2.8 \pm 1.9$ \\
\hline
\end{tabular}

At $30^{\circ} \mathrm{C}$ during rewarming, antegrade groups showed significantly higher oxygen extraction and significantly lower sagittal sinus lactate than retrograde groups $(p<0.05)$. Values are shown as mean \pm standard deviation. $A C$, Antegrade control; $R C$, retrograde control; $A E$, antegrade embolism; $R E$, retrograde embolism.

Only a small proportion of the blood from retrograde perfusion was collected in the aortic arch: between $3 \%$ and $4 \%$ in the current study, compared with $5 \%$ in our earlier work. ${ }^{15}$ Also as previously observed, the aortic arch blood collected during retrograde perfusion was desaturated. The extraction of oxygen from the retrograde perfusion return suggests that this trickle flow may contribute in some way to maintenance of metabolic function, perhaps significantly when requirements are markedly decreased by hypothermia. From the sagittal sinus lactate and oxygen extraction data during recovery, however, it is clear that retrograde flow does not support normal metabolic function as well as antegrade flow, even for a relatively short time at moderately hypothermic temperatures, as also documented by others. ${ }^{16,17}$

Nevertheless, both behavioral and histologic data suggest that a short period of moderately hypothermic retrograde perfusion is relatively benign in the absence of a concurrent insult. No animal had any clinically apparent neurologic injury after retrograde perfusion in the absence of emboli, regardless of the level of SVC pressure required to achieve adequate sagittal sinus flow rates. There is, however, evidence of cerebral injury on pathologic examination in two of five animals after retrograde perfusion, in contrast to the absence of histologic damage in any of five control animals after antegrade perfusion: the histologic damage seen in the $\mathrm{RC}$ group does not appear to correlate with high SVC pressure.

EEG recovery was also more rapid in the $\mathrm{AC}$ than in the $\mathrm{RC}$ group, confirming our previous observation that antegrade flow preserves cerebral function acutely better than retrograde flow. ${ }^{15}$ The more rapid recovery of normal values for oxygen extraction and sagittal sinus lactate content after rewarming is additional evidence of the superiority of antegrade perfusion over retrograde perfusion in maintaining optimal levels of cerebral function.

In contrast to the anticipated excellent recovery of the $\mathrm{AC}$ group, there is clear behavioral and histologic evidence of cerebral injury in the group that underwent antegrade perfusion after embolization: this demonstrates that the injection of polystyrene microspheres does cause detectable brain injury. Although average levels of behavioral impairment similar to those in the AE group were seen after embolization and retrograde perfusion in the initial $\mathrm{RE}$ group, suggesting that retrograde perfusion has no protective effect, the low mean behavioral score in the $\mathrm{RE}$ group included several animals with minor impairment and good recovery as well as some with severe and irreversible damage. Further analysis of these initial results suggested that the SVC pressure used during retrograde perfusion might correlate with outcome: the most severely affected animals, two of which died, were perfused at very high SVC pressures.

The idea that the effectiveness of retrograde perfusion in mitigating cerebral injury after embolization might depend on retrograde perfusion pressure prompted the testing of an additional 10 animals. When all 20 animals that underwent embolization and retrograde perfusion were subsequently analyzed, it became apparent that animals successfully perfused with an SVC pressure less than $40 \mathrm{~mm} \mathrm{Hg}$ show less cerebral damage after embolization than animals perfused retrograde with high pressures or animals perfused antegrade after embolization. We speculate that retrograde cerebral 
perfusion works by washing some emboli out of the cerebral circulation before irreversible tissue damage occurs, even though the difference in the number of microspheres recovered in the brains of the different groups of embolized animals in our study did not reach statistical significance. We think these positive results using retrograde perfusion at low SVC pressure after embolization are encouraging and deserve further investigation.

We are unable to explain why some pigs require much higher SVC pressure than others to achieve the same cerebral venous pressure, even in the absence of emboli. In a few pilot animals in our previous study, ${ }^{15}$ no jugular venous valves were seen angiographically. In puppies (and clinically in infants) we have observed that baseline cerebrovascular resistance is somewhat variable and that the presence of a high cerebrovascular resistance before a hypothermic ischemic insult seems to augur poorer postoperative recovery ${ }^{18}$ : it may be that the requirement of a high SVC pressure indicates an ominous propensity toward cerebral vasoconstriction. If one considers only the embolized animals, then given the increase in cerebrovascular resistance that occurs after embolization, it is possible to speculate that those animals more successfully embolized are more likely to have both higher venous resistances (requiring greater SVC pressures) and worse outcomes.

The presence of some cerebral lesions even in the $\mathrm{RC}$ animals raises the recurrent troubling question whether retrograde perfusion is potentially harmful in some individuals, as suggested by the studies of Mohri and associates ${ }^{19}$ in dogs. Although measuring sagittal sinus pressure assures that we are not attempting to perfuse the cerebral circulation at very high pressure, it is possible that the high SVC pressures necessary for retrograde perfusion in some animals may result in high pressures perfusing the brain stem and spinal cord via venous beds that do not communicate with the sagittal sinus. Although these high pressures appear to be well tolerated in control animals, it is conceivable that in combination with emboli to these regions, high pressures may produce adverse effects. In a vascular circuit blocked by an embolus or narrowed by arteriolar vasoconstriction, it is easy to imagine that high pressure could more easily provoke extravasation manifested as hemorrhage or edema and result in the increased morbidity with retrograde perfusion seen after embolization. Indeed, brain stem hemorrhage was noted in two of the animals that died, and there is experimental evidence from the work of others that high SVC pressures during retrograde perfusion are associated with increased morbidity. ${ }^{20,21}$

Once the empirical observation that poor behavioral outcome might be related to high SVC pressure during retrograde perfusion had been made, we tried to minimize the final level of SVC pressure by more slowly instituting retrograde flow. Although this strategy was not entirely successful, in that high SVC pressures were still frequently required, microsphere recovery in the brain was lower, behavioral results were much better, and histologic damage was considerably less in the last $10 \mathrm{RE}$ animals than in the earlier group, in which retrograde perfusion after embolization was instituted less gradually. Inasmuch as careful scrutiny showed no other differences in the conduct of the protocol in the two groups to explain the improved results, perhaps this technical point is important.

In this embolization protocol, in which predominantly focal rather than global ischemic injury was produced, there seems to be a slightly different relationship between histologic outcome, behavioral score, and EEG than in some of our earlier studies. ${ }^{8}$ Histologic evaluation appears to be the most sensitive technique for assessing this type of focal insult and seems to correlate better with behavioral scores early in the postoperative period. We speculate that later neurologic evaluation is less sensitive in detecting injury because healthy brain tissue gradually begins to compensate for damaged areas, resulting in improvement in behavioral scores. As in our earlier study, the level of EEG power at 2 hours after the start of rewarming is predictive of cerebral injury after antegrade or retrograde perfusion, as reflected by total histologic score, with significant differences noted between embolism and control groups.

Although we recognize that this study had some methodologic problems, it nevertheless demonstrates that some animals recover after embolization and retrograde cerebral perfusion with minimal or no cerebral injury. The complete recovery of these animals, without even histopathologic evidence of any cerebral injury, in contrast to the almost invariable neurologic impairment and histopathologic abnormalities in the AE group, adds to the existing evidence suggesting that retrograde perfusion may provide some degree of cerebral protection after embolization in the ascending aorta. Both instituting retrograde perfusion gradually and keeping SVC 
pressures below $40 \mathrm{~mm} \mathrm{Hg}$ appear to improve results. We plan to investigate this technique further.

We thank Richard Smith, Richard Henry, and Russel Jenkins for their technical assistance in the conduct of these experiments.

\section{REFERENCES}

1. Ergin MA, Griepp EB, Lansman SL, et al. Hypothermic circulatory arrest and other methods of cerebral protection. J Card Surg 1994;9:525-37.

2. Newburger JW, Jonas RA, Wernowsky G, et al. A comparison of the perioperative neurologic effects of hypothermic circulatory arrest versus low-flow cardiopulmonary bypass in infant heart surgery. $\mathrm{N}$ Engl J Med 1993;329:1057-64.

3. Bellinger DC, Jonas AR, Rapport LA, et al. Developmental and neurologic status of children after heart surgery with hypothermic circulatory arrest or lowflow cardiopulmonary bypass. N Engl J Med 1995;332: 549-55.

4. Ergin MA, Galla JD, Lansman L, et al. Hypothermic circulatory arrest in operations on the thoracic aorta: determinations of operative mortality and neurologic outcome. J THORAC CARdIOVASC SuRg 1994;107:78897.

5. Svensson LG, Crawford ES, Hess KR, et al. Deep hypothermia and circulatory arrest: determinants of stroke and early mortality in 656 patients. J THORAC CARDIOvasC SuRg 1993;106:19-31.

6. Mills NL, Ochsner JL. Massive air embolism during cardiopulmonary bypass: causes, prevention, and management. J Thorac Cardiovasc Surg 1980;80:708-17.

7. Lemole GM, Strong MD, Spagna PM, et al. Improved results for dissecting aneurysms: intraluminal sutureless prosthesis. J Thorac Cardiovasc Surg 1982;83: 249-55.

8. Mezrow CK, Midulla PS, Sadeghi AM, et al. Quantitative electroencephalography: a method to assess cerebral injury after hypothermic circulatory arrest. J Thorac Cardiovasc Surg 1995;109:925-34.

9. Deeb GM, Jenkins E, Bolling SF, et al. Retrograde cerebral perfusion during hypothermic circulatory arrest reduces neurologic morbidity. J THORAC CARDIOVASC SURG 1995;109:259-68.

10. Lin PJ, Chang C-H, Tan PPC, et al. Protection of the brain by retrograde cerebral perfusion during circulatory arrest. J ThoraC CARdIOvasC SURG 1994;108: 969-74.

11. McLoughlin TM, Carter WR, King CD. Case 2-1995: continuous retrograde perfusion as an adjunct to brain protection during deep hypothermic systemic circulatory arrest. J Cardiothorac Vasc Anesth 1995;9:205-14.

12. Pagano D, Carey JA, Patel RL, et al. Retrograde cerebral perfusion: clinical experience in emergency and elective aortic operations. Ann Thorac Surg 1995;59:393-7.

13. Safi HJ, Brein HW, Winter JN, et al. Brain protection via cerebral retrograde perfusion during aortic arch aneurysm repair. Ann Thorac Surg 1993;56:270-6.

14. Ueda Y, Miki S, Kusuhura K, et al. Deep hypothermic systemic circulatory arrest and continuous retrograde cerebral perfusion for surgery of aortic arch aneurysm. Eur J Cardiothorac Surg 1992;6:36-41.

15. Midulla PS, Gandsas A, Sadeghi AM, et al. Comparison of retrograde cerebral perfusion to antegrade cerebral perfusion and hypothermic circulatory arrest in a chronic porcine model. J Card Surg 1994;9:56075.

16. Crittenden MD, Roberts CS, Rosa L, et al. Brain protection during circulatory arrest. Ann Thorac Surg 1991;51:942-7.

17. Winsborrow BG, Kozlowski P, Filgueiras CL, et al. 31P spectroscopic imaging of pig brains during circulatory arrest. Can J Appl Spect 1994;39:135-42.

18. Mezrow CK, Gandsas A, Sadeghi AM, et al. Metabolic correlates of neurologic and behavioral injury after prolonged hypothermic circulatory arrest. J Thorac Cardiovasc Surg 1995;109:959-75.

19. Mohri H, Sadahiro M, Akimoto $\mathbf{H}$, et al. Protection of the brain during hypothermic perfusion. Ann Thorac Surg 1993;56:1493-6.

20. Nojima T, Magara T, Nakajima $Y$, et al. Optimal perfusion pressure for experimental retrograde cerebral perfusion. J Card Surg 1994;9:548-59.

21. Usui A, Oohara K, Liu T, et al. Determination of optimum retrograde cerebral perfusion conditions. J Thorac Cardiovasc Surg 1994;107:300-8.

\section{Discussion}

Dr. Roxanne Deslauriers (Winnipeg, Manitoba, Canada). Retroperfusion of the brain during deep hypothermic circulatory arrest is being used increasingly by cardiac surgeons. Although much remains to be understood about this technique, it is believed that some form of cerebral perfusion during circulatory arrest is better than no perfusion, especially if the period of arrest is prolonged. Dr. Griepp and colleagues have shown that neurologic recovery is complete after 25 minutes of arrest with either antegrade or retrograde cerebral perfusion. When the period of arrest is extended to 2 hours, large differences can be seen between the relative efficacies of antegrade and retrograde perfusion.

[Slide] This slide shows a phosphorus magnetic resonance spectrum of the pig brain, obtained by means of localized spectroscopy during bypass, circulatory arrest, and recovery. After about 40 minutes, there is loss of high-energy phosphates, which do not recover on reperfusion. When retrograde perfusion is used during circulatory arrest, there is also loss of high-energy phosphates; however, these recover on reperfusion. When antegrade perfusion is used, we find no change in the high-energy 
phosphates, either during the circulatory arrest period or during reperfusion.

Inasmuch as these long-term effects do exist, Dr. Griepp, I would like you to comment on whether a combination of antegrade and retrograde perfusion would be advisable if the period has to be prolonged and if you anticipate that there might be emboli.

Dr. Griepp. Thank you. You asked whether a combination of antegrade and retrograde perfusion may be helpful. I think most people who work in the field of congenital heart surgery or in aortic surgery believe that it is probably best to minimize the period of no perfusion or retrograde perfusion, and that is certainly our clinical approach. Nonetheless, there are some times when antegrade perfusion is not possible. On the basis of very little experimental data at this time, we think that retrograde perfusion may possibly be helpful.

In the presence of embolization, however, this pilot study would suggest that it is a double-edged sword. If high perfusion pressures are used, retrograde perfusion may actually be a detriment. This is a difficult area and the experimental design is problematic. There is still much to be learned, particularly in the presence of embolization.

Dr. Deslauriers. We find, as did Dr. Griepp, that some groups recover extremely well in terms of histopathology and some do not. I would like to suggest that we consider the use of magnetic resonance imaging to find out what goes on in the brain parenchyma of individual animals. At the moment most people are measuring either SVC pressure or superior sagittal sinus pressure, but what is actually going on in terms of perfusion within the brain remains unknown.
This could be addressed by using a combination of magnetic resonance perfusion imaging, diffusion-weighted imaging, which gives early measures of neuronal damage, and T2-weighted imaging, which gives a measure of blood oxygenation.

Dr. Griepp. I would agree. We are looking with keen interest at those of you that have the 4, 5 and $6 \mathrm{~T}$ magnets that are going to make it possible to make some of these observations. I think it is fair to say that at present we still have no direct evidence that there is flow into the parenchyma of the brain. We have preservation of high-energy phosphates, we have clinical recovery, we have absence of histopathological damage, all suggesting perfusion, but there is no direct evidence.

One further comment I would like to make is that we began this study and a previous study with the idea that if we measured sagittal sinus pressure, that would be the venous perfusion pressure of the brain during retrograde perfusion. Having seen the results of this series, we are being forced to the belief that there are probably parts of the brain drained through venous channels that are independent of one another. Although the sagittal sinus pressure may be only $30 \mathrm{~mm}$ $\mathrm{Hg}$ with a high SVC pressure, there may be other venous channels within the brain that are at pressures closer to that of the SVC. It is hard to know what is actually being perfused, and at what pressure. The variable participation of veins within the entire brain during retrograde perfusion is among the potential explanations for our findings and also among the potential hazards of using this method clinically. 